\title{
L'impegno di Aiaf Onlus per migliorare la presa in carico dei pazienti con Anderson-Fabry
}

Giornale di Tecniche Nefrologiche e Dialitiche 2019, Vol. 3I (I) 54-57

(C) The Author(s) 2019

Article reuse guidelines:

sagepub.com/journals-permissions

DOI: $10.1177 / 0394936219837494$

journals.sagepub.com/home/gtn

(S)AGE

\section{Stefania Tobaldini}

Nel giugno 2018 la nostra Associazione ha avuto l'onore di partecipare all'"Excellence Meeting I AM FABRY Accademia Malattia di Fabry", che si è svolto nella splendida cornice del Golfo di Napoli al Castel dell'Ovo. L'evento ha raccolto i maggiori esperti clinici italiani sulla Malattia di Anderson-Fabry, oltre 250 specialisti nefrologi, cardiologi, neurologi, genetisti, dermatologi e oculisti, provenienti da tutta la Penisola; alcuni di essi sono intervenuti in qualità di relatori per presentare i risultati aggiornati riguardanti i progetti clinici e di ricerca avviati negli ultimi anni. L'occasione è stata utile per confrontarsi sugli obiettivi terapeutici, sugli aspetti legati ai monitoraggi nefrologico, cardiologico e neuroradiologico, ma anche per affrontare temi inerenti la diagnostica, la genetica, l'infiammazione e lo stress ossidativo.

Per Aiaf è stata un'occasione importante per far emergere i bisogni dei pazienti di fronte ai massimi esperti italiani sulla Malattia di Fabry e ai medici che si prendono quotidianamente cura dei pazienti italiani (figura 1). Inoltre, abbiamo assistito a presentazioni di lavori scientifici di altissimo livello, abbiamo ricevuto informazioni su studi clinici in cui molti di noi pazienti erano stati coinvolti e su studi e progetti avviati a livello locale, cui vorremmo poter dare visibilità con la collaborazione e l'aiuto dei clinici coinvolti.

Nel corso del Meeting si è parlato anche di diagnosi precoce, importante per questa patologia non solo per il counseling genetico nelle forme più gravi, ma anche perché offre la possibilità di introdurre per tempo terapie specifiche che possono modificare drasticamente il corso della patologia. I segni clinici e i sintomi di questa patologia sono sfumati e di difficile identificazione, cosa che spesso comporta un grave ritardo nella diagnosi che può avere pesanti conseguenze.

"La diagnosi può diventare un'odissea durante la quale il paziente può vedere anche una decina di diversi specialisti - ha spiegato Antonio Pisani, ricercatore dell'Università Federico II di Napoli-Dall'insorgenza della malattia alla sua corretta individuazione possono passare fino a 18 anni per gli uomini e addirittura 25 per le donne! La malattia di Fabry non presenta sintomi specifici ed è ancora poco conosciuta dalla maggioranza del personale medico-sanitario. È quindi fondamentale riuscire ad aumentare il livello di conoscenza e consapevolezza tra tutti gli specialisti che possono essere coinvolti”.

Fortunatamente a questo problema è stata data una risposta con la Legge di Bilancio 2019, grazie all'approvazione di un emendamento alla L.167/2016 che ha incluso nello Screening Neonatale Esteso (reso obbligatorio per tutti i nuovi nati in Italia) anche le malattie neuromuscolari genetiche, le immunodeficienze congenite severe e le malattie da accumulo lisosomiale. Una vera e propria svolta epocale per le persone con malattia di Anderson-Fabry, che potranno così essere diagnosticate alla nascita ed avranno la possibilità di effettuare un monitoraggio precocissimo del decorso della malattia. Sarà finalmente possibile intervenire con le terapie appropriate al momento dell'effettiva necessità, evitando danni d'organo irreversibili.

Nella giornata di apertura Aiaf è intervenuta sul tema "Cosa si aspetta dal Sistema Sanitario Nazionale un paziente affetto da malattia rara nel 2018?"

Non è facile dare una risposta a questa domanda. Ogni volta che viene chiesto ad un paziente quali siano le sue aspettative, ci si sente rispondere: "una vita comune, un'esistenza normale". Questo perché convivere con una malattia rara come la malattia di Anderson-Fabry non permette di vivere in modo "comune": il tempo, per tutta la durata della vita, viene scandito da una serie di appuntamenti ospedalieri cui difficilmente ci si può sottrarre e che fanno parte del percorso di presa in carico del paziente.

Presidente Aiaf Onlus, Italy

Corrispondenza:

Stefania Tobaldini, Presidente Aiaf Onlus.

Email: presidente@aiaf-onlus.org 


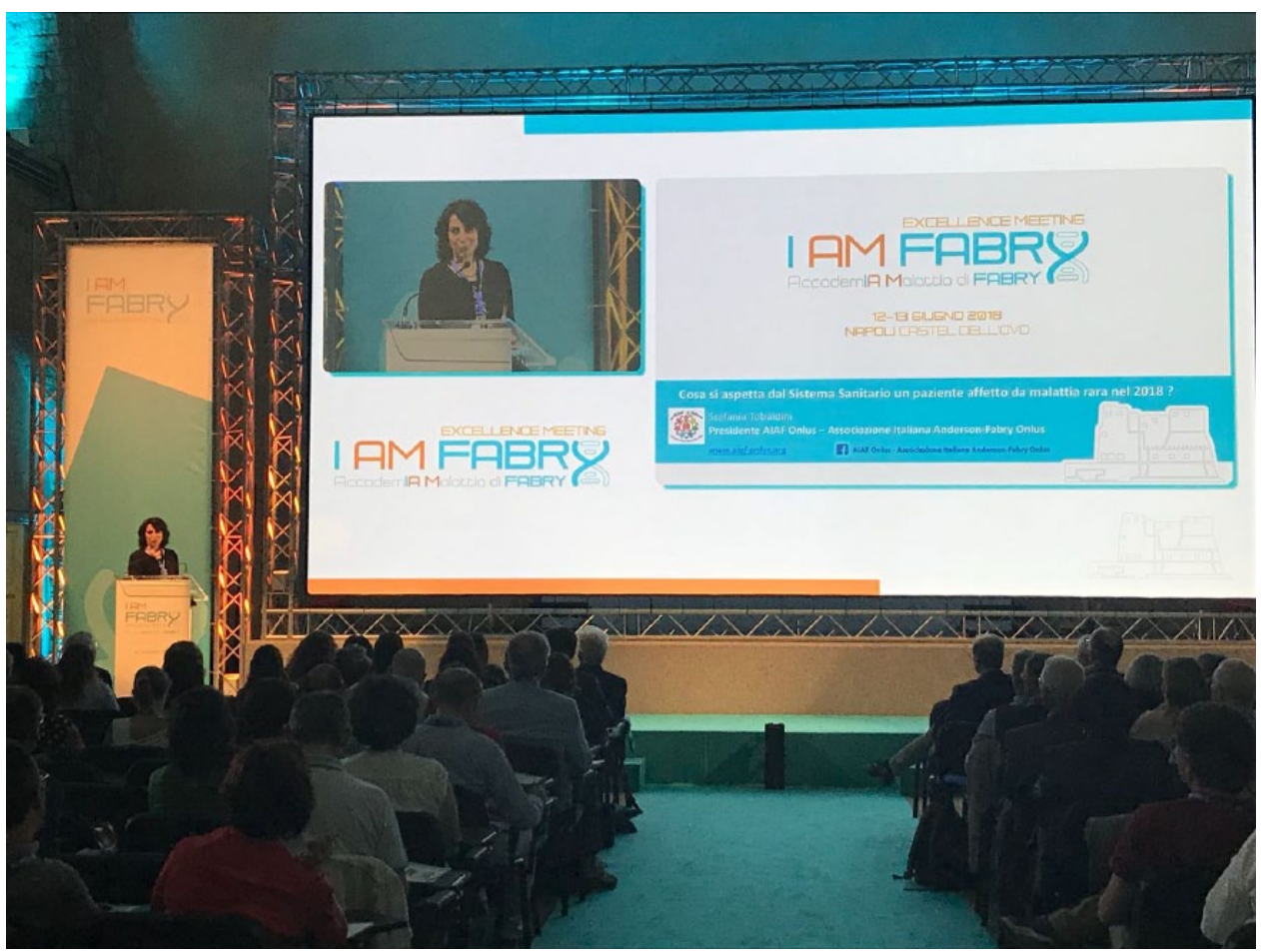

Figura I. Un momento del Convegno "I AM Fabry” - Napoli.

La presa in carico del paziente è caratterizzata da alcuni momenti significativi e per questo potrebbe essere sintetizzata in "tappe", ognuna delle quali porta con sé un bagaglio di aspettative da parte del paziente, ma anche di conseguenze: la diagnosi, la comunicazione della diagnosi e il follow-up.

La presa in carico di un paziente inizia dal momento della diagnosi (che, come già detto, spesso per i pazienti Anderson-Fabry tarda ad arrivare a causa della complessità della sintomatologia), rappresentando però solo un punto di inizio: a partire da quel momento, la nuova famiglia entra in contatto con una "malattia rara", una situazione nuova, spiazzante e potenzialmente devastante per dei neogenitori (nel caso si tratti di diagnosi neonatale) o per il paziente (nel caso sia tardiva).

Nel caso di diagnosi neonatale, è importante, prima ancora della diagnosi, un corretto, chiaro e onesto processo informativo, che venga avviato già a partire dai percorsi di preparazione al parto per evitare gli effetti negativi di una scarsa consapevolezza o di una scarsa comprensione dell'argomento.

Ricordiamo poi che la comunicazione della diagnosi è un momento molto delicato, che può creare un trauma al paziente e/o ai genitori del paziente pediatrico: se non viene affrontata adeguatamente, rischia di trascinare nella "trappola" della paura, del sospetto, del timore di un'ingerenza nella sfera personale. A tutti gli effetti questo momento cambierà per sempre la vita di un'intera famiglia, che probabilmente dovrà confrontarsi anche con la necessità di effettuare uno screening familiare, con la conseguente conferma della diagnosi anche per uno dei genitori, per i figli e forse per qualche altro parente, trattandosi di una malattia genetica ereditaria. Una diagnosi di malattia rara può portare una famiglia all'isolamento e al rifiuto di una condizione "non voluta".

Anche la comunicazione di un sospetto di diagnosi è un momento estremamente delicato: oggi vengono condotti alcuni screening mirati tra $\mathrm{i}$ pazienti con malattie renali, con cardiomiopatie o con problemi che interessano il sistema nervoso centrale, ed accade spesso che non venga fornita loro un'adeguata ed esaustiva informazione sulla malattia. Con il risultato che il paziente, in attesa del risultato del test, si trova disorientato e spaventato. Sempre più spesso l'Associazione Pazienti viene trovata attraverso ricerche autonome sul web da persone che vivono con preoccupazione l'attesa di una conferma di diagnosi, e sono alla ricerca di informazioni via web sui sintomi, sull'aspettativa di vita e sulle possibili cure.

La comunicazione medico-paziente è dunque fondamentale. Oggi i pazienti e le loro famiglie sono molto esigenti, vogliono essere "parte attiva" e spesso vanno alla ricerca spasmodica di informazioni sul web. Purtroppo, però, in rete non sempre si trovano notizie corrette. Una chiara e dettagliata informazione da parte del medico di riferimento può evitare questo pellegrinaggio in rete e può quindi aiutare la famiglia e il paziente ad acquisire una maggiore consapevolezza sulla patologia. Anche il contatto con l'Associazione Pazienti di riferimento (a cui 
auspichiamo i pazienti vengano indirizzati dai medici) può favorire il processo di acquisizione di consapevolezza ed accettazione della condizione di "rarità".

Dopo la diagnosi di una malattia multisistemica, come è la Malattia di Anderson-Fabry, è necessario che il paziente venga preso in carico da un'equipe multidisciplinare. Sarà pertanto fondamentale che la famiglia venga indirizzata ad un Centro di Riferimento Regionale Accreditato per il monitoraggio dell'andamento della malattia.

Attualmente, purtroppo, la presa in carico presenta diverse criticità, dovute alla scarsa numerosità dei pazienti, all'assenza di multidisciplinarietà all'interno di alcuni Centri di Riferimento, alle difficoltà organizzative e all'assenza di un PDTA (Percorso Diagnostico Terapeutico Assistenziale) o di modelli omogenei di presa in carico sul territorio nazionale. Per questi motivi, spesso il paziente si trova disorientato ed è costretto a spostarsi al di fuori della propria regione di residenza, per poter effettuare $\mathrm{i}$ monitoraggi con una certa regolarità.

Spesso il paziente si trova a vivere le incertezze su quale sia il momento opportuno per iniziare una terapia, oppure non comprende per quale motivo non gli sia stata prescritta una terapia, oppure ancora trova difficoltà a conciliare la propria vita con la gestione della malattia, delle terapie e delle visite di controllo, per le quali è costretto ad assentarsi ripetutamente dal lavoro o da scuola. Trattandosi di una malattia multisistemica, il paziente deve infatti sottoporsi regolarmente ad una serie di esami strumentali e visite di controllo multispecialistiche, che difficilmente possono essere organizzate in un'unica giornata e per le quali si rendono necessari più accessi ospedalieri in giorni separati per completare gli accertamenti di monitoraggio. Con evidenti disagi e difficoltà nel condurre una vita "normale", al punto che a volte il paziente si sente scoraggiato nell'effettuare con regolarità le visite di controllo. A complicare la situazione, c'è anche il fatto che, a volte, i pazienti si trovano a fare i conti con le disparità regionali per quanto riguarda la domiciliazione delle terapie, permesse solo in alcune regioni in Italia. Tutto questo, soprattutto per i pazienti in terapia enzimatica sostitutiva residenti in alcune regioni, comporta un numero considerevole di accessi in ospedale nel corso dell'anno, difficilmente giustificabili per chi ha un lavoro.

Una gestione frammentata degli appuntamenti per il follow-up, cui si somma la distanza geografica tra l'abitazione del paziente e il Centro di Riferimento, rischia pertanto di diventare per il paziente non solo una spesa, ma anche un ulteriore motivo di stress e di difficoltà. E questo, ricordiamolo, in assenza di tutele specifiche in ambito lavorativo dovute al mancato riconoscimento di invalidità da pare delle commissioni INPS, molto frequente per i pazienti affetti da una malattia poco conosciuta come la malattia di Anderson-Fabry.
La transizione dall'età pediatrica all'età adulta è inoltre una fase molto particolare, perché coincide con l'età adolescenziale in cui il giovane paziente può mettere in discussione l'opportunità di prendersi cura della propria salute. È importante gestire questo passaggio in modo rassicurante per le famiglie e per i giovani pazienti, con gradualità $\mathrm{e}$ individuando all'interno dell'equipe una figura di riferimento dedicata.

Per queste, ma anche per altre situazioni, sarebbe inoltre auspicabile un adeguato supporto psicologico che accompagni il paziente lungo tutto il suo percorso di presa in carico, per una piena accettazione e consapevolezza della propria condizione di rarità, anche se spesso questo aspetto viene sottovalutato; proprio per questo lo psicologo non è previsto all'interno dell'equipe multidisciplinare.

Altre problematiche irrisolte relative alla presa in carico della persona nella sua globalità, sono il mancato collegamento tra il medico di Medicina Generale (o pediatra di Libera Scelta), il Centro di Riferimento e la gestione territoriale delle esigenze assistenziali del paziente: spesso spetta alla famiglia farsi carico della ricerca della soluzione più appropriata, e a proprie spese, nei casi di compromissioni gravi in cui sia necessario un percorso di riabilitazione.

Tutte queste considerazioni, che rendono più difficile per il paziente sostenere la sua patologia nella quotidianità, hanno portato Aiaf ad interrogarsi sulla possibilità di cercare soluzioni in grado di ottimizzare la presa in carico delle persone con malattia di AndersonFabry.

Oltre a lavorare affinché in ogni regione in Italia sia possibile l'accesso alla terapia domiciliare (per i pazienti considerati idonei dallo specialista di riferimento), da qualche mese Aiaf ha anche avviato un nuovo progetto "Caring Fabry", in collaborazione con un'agenzia specializzata ed alcuni Centri ospedalieri di Riferimento italiani di eccellenza. Obiettivo: delineare un modello ideale di presa in carico dei pazienti che permetta di integrare il PDTA e la Gestione Integrata della malattia.

Partendo dall'esame dell'esperienza di alcuni Centri di eccellenza italiani, con i quali ha avviato una collaborazione, e con il supporto del proprio Comitato Scientifico, Aiaf si propone di elaborare un modello in cui sia definito in maniera puntuale e concreta la cadenza temporale con la quale il team multidisciplinare (composto da tutti gli specialisti degli organi che sono interessati dalla patologia) deve confrontarsi per discutere i singoli casi (nuovi o follow-up), come far dialogare anche a distanza i diversi professionisti per assicurare la gestione multidisciplinare del singolo caso, quali esami di controllo e con quale periodicità andranno svolti, quale figura professionale deve occuparsi della presa in carico del singolo paziente e 


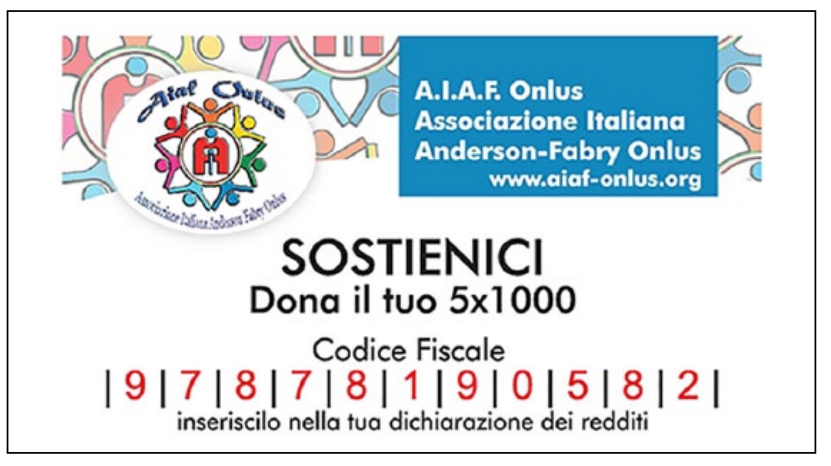

con quale strumentazione informatica fissare e gestire le prenotazioni e le informazioni per il paziente. Infine, ci si propone di delineare le modalità con cui il Sistema Sanitario regionale potrà facilitare il rispetto dei controlli previsti senza aspettare l'iniziativa del singolo paziente e i modi e gli strumenti che ne facilitino il percorso, eliminando vincoli burocratici ed appesantimenti logistici.

$\mathrm{Ci}$ auguriamo che il progetto possa portare ad un'ottimizzazione delle risorse, ad una migliore gestione del tempo e al miglioramento della qualità della vita dei pazienti.

\section{MEETING NAZIONALE 2019}

Save

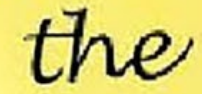

Date

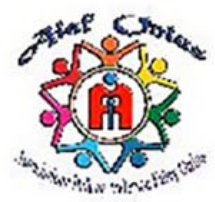

23 - 24 MARZO 2019

Rimini - Hotel Savoia

Viale Lungomare Murri 13

\section{Vi aspettiamo !}

Per informazioni su come partecipare inviare una mail a: presidente@aiaf-onlus.org 